\title{
CONVERGENCE ANALYSIS OF THE ITERATIVE METHODS FOR QUASI COMPLEMENTARITY PROBLEMS
}

\author{
MUHAMMAD ASLAM NOOR \\ Department of Mathematics \\ College of Science, King Saud University \\ Riyadh 11451, Saudi Arabia.
}

(Received November 17, 1986)

ABSTRACT. In this paper, we consider the iterative methods for the quasi complementarity problems of the form

$$
u-m(u)>0, \quad T(u) \geq 0, \quad(u-m(u), T(u))=0,
$$

where $m$ is a point-to-point mapping and $T$ is a continuous mapping from $\mathbf{R}^{\mathbf{n}}$ into itself. The algorithms considered in this paper are general and unified ones, which include many existing algorithms as special cases for solving the complementrity problems.

KEY WORDS AND PHRASES. Quasi complementarity problem, quasi variational inequality, convergence, iterative methods, algorithms.

1980 AMS (MOS) SUBJECT CLASSIFICATION CODE. 49A29, 73C60, 65K05, 90C33.

\section{INTRODUCTION.}

variational inequalities theory introduced by stampacchia and Fichera in 1964, and later developed by the French and Italian schools, has enjoyed a vigorous growth for the last twenty years. Variational inequality theory not only reveals the fundamental facts on the qualitative behaviour of solutions (regarding existence, uniqueness and regularity) to important classes of nonlinear boundary value problems, but also provides highly efficient new numerical methods to solve for example free and moving boundary value problems. In brief, it is now clear that the theory of variational inequalities provides a natural and elegant framework for the study of many seeiningly unrelated free boundary value problems arising in fluid flow through porous media, contact problems in elasticity, operations research, economics and transportation equilibrium etc.

Equally important is the area of mathematical programming known as complementarity theory. This area of operations research is a relatively new one and one which has received much attention during the last two decades. The impetus behind the identification of the complementarity problem in the early 1960's was the Kuhn-Tucker conditions for nonlinear programming which give necessary conditions of

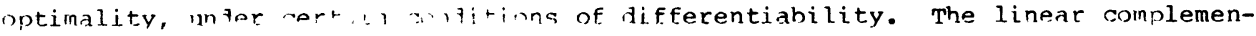
tarity problem was introduced by wenke in 1964, but it were cottle and Dantziq [1], who formally defined the linear coinplementarity problem and called it the fundamental problem. A survey paper by Lemke [2] outlines the early theoretical results, most of 
which were inotivated by applications to equilibrium type problems in operations research and game theory. For most recent results and applications, see Bensoussan and Lions [3], Crank [4], Baiocchi and Capelo [5], Oden and Kikuchi [6], and the references therein. An important and useful generalization of the variational inequality problem is the quasi variational inequality problem, introduced and studied by Bensoussan and Lions [3] in the impulse control. For other applications of quasi variational inequalities, see $[4,5,6]$ and the references therein. The complementarity problem has also been extended by Habetler and Price $[7,8]$, and Karamardian [9]. Dolcetta [10] has considered the implicit (quasi) complementarity problem, which is an another generalization of complementarity problem. The relationship between a variational inequality problem and a complementarity problem has been noted implicity by Lions [11] and Mancino and Stampacchia [12]. However, it was Karamardian $[9,13]$, who showed that if the set involved in a variational inequality problem and complementarity problem is a convex cone, then both problems are equivalent. It has been shown by Pang $[14,15]$, and Noor [16] that such a relationship is preserved in both the quasi variational inequality problem and the quasi complementarity problem. This equivalence plays a fundamental and central part in suggesting new and unified iterative algorithms for solving complementarity problem and its various generalizations. Noor [17] and Chan and Pang [18] have shown that the solution of a class of quasi variational inequalities can be obtained from an iterative scheme, which is obtained by using the projection technique.

Inspired and motivated by the recent research work going on in these fields, we propose and analyze a new and unified algorithm for the quasi complementarity problems. Most of the convergence properties of the Mangasarian's algorithm discussed in $[14,15,19]$ are carried over to this new proposed algorithm. For related work, see pang [15], where he has used the least element theory and contraction type arguments based on the splitting of the underlying matrix to prove the existence of the solution. In this paper, we use the variational inequality technique to study the convergence properties of the suggested algorithm for both the linear and nonlinear cases. Our results are an extension and improvement of the results of Noor and Zarae [20], Pang [14], Ahn $[21,22]$ and Mangasarian [19].

In section 2, after reviewing some basic notations and facts, we introduce the quasi complementarity problem and discuss several special cases. Algorithms and convergence results are discussed and derived in sections 3 and 4.

\section{PRELIMINARIES AND BASIC RESULTS.}

We denote the inner product and norm on $R^{n}$ by $(\ldots$, ) and $\|$.$\| , respectively.$ Let $C$ be a closed convex set in $R^{n}$.

Given a continuous mapping $T$ from a convex set $C$ in $R^{n}$ into $R^{n}$, we consider the problem of finding $u \in C$ such that

$$
(T(u), v-u)>0, \quad \text { for all vec. }
$$

This type of problem is known as variational inequality problem, introduced by Stampacchia [23] and Fichera [24]. Note that if $C=\mathbf{R}^{n}$, then problem (2.1) is equivalent to finding $u \in R^{n}$ such that

$$
(T(u), v)=0, \quad \text { for all } v \varepsilon_{R^{n}}
$$


problein (2.2) is known as the weak formulation of boundary value problems, where $T$ is any differential or integral operator associated with the qiven problem, see [5, 11]. If $C$ is a convex cone in $R^{n}$, then problem (2.1) is erquivalent to finting uec such that

$$
(T(u), v) \geq 0, \text { and }(T(u), u)=0, \text { for all vec. }
$$

This observation is lue to Lions [11] and Mancino and Stampacchia [12].

Furthermore, we note that for some elements $v$, equality holds in the variational inequality (2.1). This happens, when together with $v, 2 u-v$ also lies in $c$. In this case, problem $(2.1)$ is equivalent to finding uEc such that

$$
(T(u), v-u)=0,
$$

Mosco and Strany [25] have pointed out that if $u$ is not an extreme point of the convex set $C$, then there are directions in which $u$ is interior to a line segment and in these directions the equality holds. A similar conclusion has been drawn by Mancino and Stampacchia [12] and Noor [26].

Lions and Stampacchia [27], and Glowinski, Lions and Tremolieres [28] used the projection technique to prove the existence of a unique solution of the variational inequality $(2.1)$. In 1979, Noor and Noor $[29,30]$ proved that the solution of the variational inequality $(2.1)$ can be obtained by an iterative scheme namely:

$$
u_{n+1}=P_{C}\left[u_{n}-\rho T\left(u_{n}\right)\right], \quad n=0,1,2, \ldots
$$

where $\rho>0$ is a constant and $P_{C}$ is the projection of $R^{n}$ into $C$ and studied the convergence criteria.

In the formulation of the variational inequality, the underlying convex set $c$ does not depend upon the solution. In many applications, the convex set also depends implicity on the solution $u$ itself. In this case, the variational inequality (2.1) is known as quasi variational inequality. To be more specific, a quasi variational inequality problem is indeed a problem of the type:

Given a point-to-set mapping $c: u \longrightarrow c(u)$, which associates a closed convex subset $C(u)$ of $R^{n}$ with any element $u$ of $R^{n}$, find $u \varepsilon c(u)$ such that

$$
(T(u), v-u) \geq 0, \quad \text { for all } v \varepsilon C(u) \text {. }
$$

In many important applications, see Mosco [31], Bensoussan and Lions [3], and Baiocchi and Capelo [5], the set $C(u)$ is of the following form:

$$
c(u)=m(u)+C
$$

where $m$ is a point-to-point mapping and $c$ is a closed convex set in $R^{n}$. Note that if the point-to-point mapping $m$ is zero, then the quasi variational inequality problem is exactly the variational inequality problem (2.1).

Related to the quasi variational inequality problem, there is a quasi complementarity problem. For a given continuous mapping $T$ from $\mathbf{R}^{\mathbf{n}}$ into itself and a point-topoint mapping $m$, we consider the problem of finding $u \varepsilon c(u)$ such that 


$$
u-m(u)>0, \quad T(u)>0, \quad(u-m(u), \quad T(u))=0,
$$

The problen $(2.8)$ is known as the quasi complementarity problem, studied by Dolecetta $[10]$, Pang $[14,15]$ and Noor and Zarae $[20]$. If the mapping $T$ is nonlinear, then we call it nonlinear quasi complementarity problem, which has been considered and studied by Noor [32]. If the mapping $T$ is an affine transformation of the form $T: u \longrightarrow M M+q$, for $M \in R^{n \times n}$ a matrix and $q \in R^{n}$ a vector, then problem (2.8) is equivalent to finding $u$ such that

$$
u-m(u)>0, \quad M u+q \geq 0, \quad(u-m(u), \quad M u+q)=0,
$$

which is a linear quasi complementarity problem. problem of type (2.9) has been studied by Dolcetta [10], where it arises as a discretized version of variational inequalities. Pang $[14,15]$ and Noor and zarae [20] studied a convergence theory for a certain type of iterative algorithms to solve (2.9) and showed that their theories unified and extended many previous convergence results for the successive-overrelaxation method for solving (2.9). The basic device employed throughout pang's work is a certain implicitly defined mapping, which is constructed from the splitting of the matrix $M$, whereas Noor and zarae [20] used the technique of variational inequalities mainly developed by Noor and Noor $[29,30]$.

If the point-to-point mapping $m$ is zero, then problem $(2.9)$ is equivalent to finding $u$ such that

$$
u \geq 0, \quad M u+q>0, \quad(u, M u+q)=0,
$$

which is known as the linear complementarity problem, originally introduced by Lemke [2] and Cottle and Dantzig [1], and then studied by Mangasarian [19], Ahn [19,22], Aganagic [33] and Pang [14] by using the iterative methods. For the applications of these complementarity problems in general equalibrium theory, free boundary value problems, and operations research, see Cottle [34], Ahn [22] and Crank [4].

Let $C$ be a convex cone with its polar $c^{*}$ defined by $c^{*}=\left\{u \varepsilon R^{n},(u, v)>0\right.$ for all $v \varepsilon c\}$. We now consider the generalized quasi complementarity problem of finding $u \varepsilon C(u)$ such that

$$
T(u) \varepsilon C^{*}(u), \quad(u, T(u))=0,
$$

where $C^{\star}(u)$ is the polar cone of $c(u)$. The generalized quasi complementarity problem was introduced and studied by Noor [16] along with the convergence criteria.

Furthermore, if the point-to-point mapping $m$ is zero, then problems (2.8) and (2.11) are equivalent to finding $u$ such that

$$
u>0, \quad T(u) \geq 0, \quad(u, T(u))=0
$$

and

$$
\text { ueC, } \quad T(u) \varepsilon C^{\star}, \quad(u, T(u))=0,
$$

which are known as nonlinear complementarity problem and generalized nonlinear complementarity problem respectively studied by cottle [34],Fang [35], Aganagic [33], Karamardian [9], Habetler and Price [7]. 


\section{ALGORITHMS .}

we need the following results, the first one is a generalization of pany [15] and Karanardian [9].

Lemma 3.1. If $C$ is a convex cone in $\mathbf{R}^{n}$, then $u \in C$ is a solution of the quasi varıational inequality $(2.6)$ if and only if uec solves the quasi complementarity problem (2.8), where $C$ is defined by the relation ( 2.7$)$.

PROOF. Suppose that a solves $(2.6)$. Since $C(u)=m(u)+C$, so vec(u) can be written as $v=m(u)+z$, for all zEC. Now

$$
\begin{aligned}
(T(u), v-u) & =(T(u), m(u)+z-u) \\
& =(T(u), m(u)-u)+(T(u), z) \\
& =(T(u), z), \quad \text { since }(T(u), m(u)-u)=0 . \\
& >0, \quad \text { since } T(u) \varepsilon C^{\star}(u) .
\end{aligned}
$$

Thus it follows that if $u$ solves the problem (2.6), u also satisfies the guasi variational inequality $(2.8)$.

Conversely suppose that $u \varepsilon C(u)$ satisfies (2.8), then clearly $u-m(u) \varepsilon C$, which implies that $2(\mathrm{u}-\mathrm{m}(\mathrm{u})) \varepsilon \mathrm{C}$, since $C$ is a convex cone by assumption. Also $0 \varepsilon c$ implis that $m(u) \varepsilon C(u)$. Taking $v=2(u-m(u))$ and $v=m(u)$ in (2.8), we obtain

$$
(\mathrm{T}(\mathrm{u}), \mathrm{u}-\mathrm{m}(\mathrm{u}))>0
$$

and

from which, it follows that

$$
(T(u), u-m(u))<0,
$$

$$
(T(u), m(u)-u)=0,
$$

Taking $v=m(u)+z$, for all $z \varepsilon C$ in $(2.8)$, we obtain

$$
\begin{aligned}
o<(T(u), v-u) & =(T(u), m(u)+z-u) \\
& =(T(u), z), \text { by }(3,1) .
\end{aligned}
$$

This implies that $T(u) \varepsilon C^{\star}(u)$. Hence $u \varepsilon C(u)$ satisfying (2.8) is also a solution of $(2.6)$, which is the required result.

Lemma 3.2 [14.17]. If $\mathrm{C}(\mathrm{u})$ is defined by relation $(2.7)$, then $u \varepsilon C(u)$ is a solution of quasi variational inequality (2.6) if and only if $u$ satisfies the following relation.

$$
u=m(u)+P_{C}[u-\rho T(u)-m(u)],
$$

for some $\rho>0$. Here $P_{C}$ is a projection of $R^{n}$ into $C$ and $m$ is a point-to-point mappinq. Special cases.

(1). If the point-to-point mapping $m$ is zero, then lemma 3.1 is exactly the same as proved by Karamardian [9] and cottle [34]. Furthermore, lemma 3.2 reduces to a result of Noor and Noor [29] for a class of variational inequalities.

(2). If $C(u)$ is equal to the non-negative orthant $R_{+}^{n}=\left\{u \varepsilon R^{n} ; u>0\right\}$, for all $u$, then lemma 3.1 reduces to a result of Pang [15], for linear quasi complementarity problem (2.9). 
Remark 3.1. It is obvious that lemma 3.1 implies that if the convex set involved in both the quasi variational inequality and the quasi complementarity problem is a convex cone, then botil the problems are equivalent, whereas lemma 3.2 together with lemma 3.1 shows that the quasi complementarity problem (2.8) can be transformed to a fixed point problem of solving

$$
u=f(u)
$$

where

$$
f(u)=\lambda m(u)+\lambda P_{C}[u-\rho T(u)-m(u)]+(1-\lambda) u,
$$

with a positive constant $\rho$ and a relaxation parameter $0<\lambda \leqslant 1$ used after projection, see [19] for more details.

Based on these observations, we now propose the following general and unified algorithm for the quasi complementarity problem (2.8).

ALGORITHM 3.1. For any given $u_{0} \varepsilon C$, compute;

$$
u_{n+1}=\lambda m\left(u_{n}\right)+\lambda P_{C}\left[u_{n}-\rho T\left(u_{n}\right)-m\left(u_{n}\right)\right]+(1-\lambda) u_{n}, n=0,1, \ldots
$$

where $\rho>0$, and $0<\lambda<1$.

If $T$ is a linear transformation of the type $T: u \longrightarrow M u+q$, for $M R_{R}^{n \times n}$ and $q \in R^{n}$, then algorithm 3.1 can be written in the following form:

ALGORITHM 3.2. For any given $u_{0} \varepsilon C$, compute

$u_{n+1}=\lambda\left(m\left(u_{n}\right)+\lambda P_{C}\left[u_{n}-\rho E_{n}\left\{u_{n}+q+I_{n}\left(u_{n+1}-u_{n}\right)\right\}-m\left(u_{n}\right)\right]+(1-\lambda) u_{n}, \quad n=0,1,2, \ldots\right.$

where $0<\lambda<1, p>0$ a constant, $\left\{E_{n}\right\}$ and $\left\{L_{n}\right\}$ are bounded sequencies of matrices in $\mathbf{R}^{\mathrm{n} \times \mathrm{n}}$. This algorithm is compatible with the algorithm of Mangasarian [19].

For the above alqorithm to be practical, $I_{n}$ may be strictly lower or upper triangular matrix, because the iterate $u_{n+1}$ may be obtained by solving variational inequality subproblem as pointed out in Pang [14]. Here the original data M remains intact throughout iteration, allowing this algorithm to be efficient both for large scale and specially structured problems.

For simplicity, we consider the case $E_{n}=E$ and $L_{n}=L$. We here consider the following version of algorithm 3.2 for linear quasi complementarity problem (2.9).

ALGORITHM 3.3. For any given $u_{0} \varepsilon C$, compute

$$
u_{n+1}=\lambda m\left(u_{n}\right)+\lambda P_{C}\left[u_{n}-\rho E\left\{M u_{n}+q+L\left(u_{n+1}-u_{n}\right)\right\}-m\left(u_{n}\right)\right]+(1-\lambda) u_{n}, n=0,1,2, \ldots
$$

where $\rho>0$ is a constant and $0<\lambda<1$ is a relaxation parameter used after the projection.

It is clear that each iteration of algorithms $3.1,3.2$ and 3.3 is itself equivalent to variational inequality problem as implied by lemma 3.1 .

\section{Special Cases 3.2 .}

1. If the point-to-point mapping $m$ is zero, then Algorithm 3.1 and Algorithm 3.2 reduce to the algorithms of Noor [41] and Ahn [22] respectively. 
ALGORITHM 3.4 [41]. Let $u_{0} \varepsilon C_{-}$, then compute

$$
i_{n+1}=(1-\lambda) u_{n}+\lambda P C\left[u_{n}-\rho T\left(u_{n}\right)\right], \quad n=0,1,2, \ldots
$$

where $0<\lambda<1$ and $\rho>0$ is a constant.

ALGORITHM 3.5 [22]. For given $u_{0} \varepsilon C$, compute

$$
u_{n+1}=(1-\lambda) u_{n}+\lambda P_{C}\left[u_{n}-\rho E_{n}\left\{u_{n}+\left(q+L L_{n}\left(u_{n+1}-u_{n}\right)\right\}\right], n=0,1,2, \ldots\right.
$$

where $\lambda<\lambda \leqslant 1$ and $\rho>0$ is a constant.

Ahn [22] has established the convergence criteria of Algorithm 3.5 for both the symmetris ani non-symmetric natrix M.

2. If $\lambda=1$, then algorithm 3.3 and alqorithm 3.1 are exactly the same as studied and andlyzed by Noor and Zarae [20], and Noor [32] respectively.

AOGORITHM $3.6[20]$. For given $u_{0} \varepsilon C$, compute

$$
u_{n+1}=m\left(u_{n}\right)+2_{C}\left[u_{n}-p E\left\{u_{n}+q+L\left(u_{n+1}-u_{n}\right)\right\}-m\left(u_{n}\right)\right], \text { for } n=0,1,2, \ldots
$$

where $\rho>0$ is a constant.

ALGORITHM $3.7[32]$. For given $u_{0} \varepsilon C$, compute

$$
u_{n+1}=m\left(u_{n}\right)+P_{C}\left[u_{n}-\rho T\left(u_{n}\right)-m\left(u_{n}\right)\right], \quad n=0,1,2, \ldots
$$

with $\rho>n$ is a constant.

Noor and Zarae [20] have studied the convergence criteria of algorithm 3.6 for both symmetric and non-symmetric matrix M.

3. If the mapping $m$ is zero and $C=R_{+}^{n}$, then algorithms 3.2 and 3.6 are exactly the same as studied and analyzed by Mangasarian [19] and Ahn [21] respectively.

ALGORITHM $3.8[19]$. For given $u_{0}>0$, compute

$$
u_{n+1}=(1-\lambda) u_{n}+\lambda\left[u_{n}-\rho E_{n}\left\{M u_{n}+q+L_{n}\left(u_{n+1}-u_{n}\right)\right\}\right]_{+}, \quad n=0,1,2, \ldots
$$

for $0<\lambda<1$ and $\rho>0$, a constant.

ALGORITHM 3.9 [21]. For given $u_{0}>0$, calculate

$$
u_{n+1}=\left[u_{n}-p E\left\{M_{n}+q+L\left(u_{n+1}-u_{n}\right)\right\}\right]_{+}, \quad n=0,1,2, \ldots
$$

Concerning the convergence of the alqorithm 3.8, Mangasarian [19] established a general convergence result under the crucial assumption that $M$ is symmetric. The result asserts that there exist accumulation points of the sequence generated by alqorithm 3.8 , and each point solves the linear complementarity problem

$$
u>0, \quad M u+q>0, \quad(u, M u+q)=0
$$

It was Ahn [21], who proved that, if the matrix $M$ is nonsymmetric, then the sequences generated by Algorithms 3.5 and 3.9 converges to the unique solution of $(3.6)$. 
4. We also note that by taking $L_{n}=0, E_{n}=I, \lambda=1$ and the point-to-point mapping $m$ equal to zero, the algorithin 3.2 reduces to the algorithm of Aganagic [33], where he has studied the convergence of this algorithm.

ALGORITHM 3.10 [33]. For given $u_{0} \varepsilon C$, conpute

$$
u_{n+1}=P_{C}\left[u_{n}-p\left(M_{n}+q\right)\right], \quad n=0,1,2, \ldots
$$

For the corresponding variational inequalities, these algorithms are mainly due to Noor and Noor [29] and Noor [36], where they have discussed the convergence criteria and applications in infinite dimensional spaces.

From the above discussion, it is clear that Algorithms $3.1,3.2$ and 3.3 proposed in this paper are nore general and include many previously known algorithms as special cases, which are mainly due to cryer [37], Aganagic [33], Cottle and Goheen [38], Ahn $[21,22]$, Chan and pang [18] and Pang [14].

4. CONVERGENCE NNALYSIS. In this section, the convergence criteria of the approximate solution obtained bfy the suggested algorithms 3.1 , and 3.3 are studied. For the algorithm 3.3, we only consider the special case, when $c=[0, b]$ is a closed convex set in $\mathbf{R}^{n}$. In this case, we consider the projection operator $P_{C^{\prime}}$ which is defined as

$$
P_{C}(u)=\arg \min _{v \in C}|| v-u|| \text {. }
$$

If $C=R_{+}^{n}$, then

$$
\left(P_{C}(u)\right)_{i}=\max \left\{0, u_{i}\right\}, \quad i=1,2, \ldots, n \text {. }
$$

In our case, we have

$$
\begin{aligned}
{ }_{C}^{\left(P_{C}(u)\right)_{i}} & =\left(P_{[0, b]}{ }^{(u))_{i}}\right. \\
& =\min \left\{\max \left(0, u_{i}\right), b_{i}\right\}, \quad i=1,2, \ldots, n .
\end{aligned}
$$

For notational purpose, ${ }^{P_{[0, b]}}$ will be denoted as $P_{C}$. The operator $P_{C}$ has the following properties, see Ahn [22].

Lemma 4.1. For any $u$ and $v$ in $R^{n}$,

(i) $u<v$ implies $P_{C}(u)<P_{C}(v)$

(ii) $P_{C}(u)-P_{C}(v)<P_{C}(u-v)$

(iii) $P_{C}(u+v)<P_{C}(u)+P_{C}(v)$

(iv) $P_{C}(u)+P_{C}(-u)<|u|$; with equality, if and only if $-b<u<b$.

In addition, we also need the following concepts. A real matrix $\operatorname{MER}^{\mathrm{n} \times \mathbf{n}}$ is said to be z-matrix (a p-matrix), if it has non-positive off-diagonal entries (positive principal minors). A square matrix with non-positive off-diagonal elements and with $\rightarrow$ non-negative inverse is called an M-matrix. It can be shown that a matrix which is

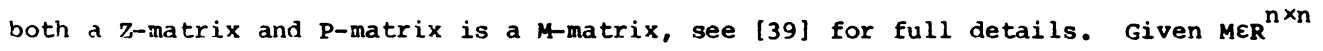
we define its comparison matrix

$$
{ }^{M} C=\left(C_{i j}\right) \text { by } c_{i i}=\left|M_{i i}\right| \text { and } c_{i j}=-\left|M_{i j}\right|, i \neq j, \quad i j=1,2, \ldots, n \text {. }
$$


If $M \varepsilon R^{m \times n}$, then $|M|$ denotes the matrix obtained from $M$ by replacing each element $M_{i j}$ by its absolute value.

The convergence of the sequences $\left\{u_{n+1}\right\}$ generated by algorithms 3.1 and 3.3 are now studied. We modify the technique of ahn [22] to prove the main results of this section.

THEOREM 4.1. Suppose that there exists a non-negative matrix NER ${ }^{n \times n}$ such that

$$
|m(u)-m(v)|<N|u-v|, \quad \text { for all } u, v \text {. }
$$

If $\left\{u_{n+1}\right\}$ and $\left\{u_{n}\right\}$ are the sequences generated by Algorithm 3.3 then

$$
\left|u_{n+1}-u_{n}\right| K(I-\lambda \rho E|L|)^{-1}[2 \lambda N+|I-\lambda \rho E(M-L)|]\left|u_{n}-u_{n-1}\right| \text {. }
$$

and

$$
\left|u_{n+1}-u\right|<(I-\lambda \rho E|L|)^{-1}[2 \lambda N+|I-\lambda \rho E(M-L)|]\left|u_{n}-u\right|
$$

for each $n$ and $u$ is the solution of linear quasi complementarity problem (2.9).

PROOF. From algorithm 3.3 , we have

$$
\begin{aligned}
u_{n+1}-u_{n}=\lambda m\left(u_{n}\right)-\lambda m\left(u_{n-1}\right)+\lambda P_{C}\left[u_{n}-\rho E\left\{M_{n}+q+L\left(u_{n+1}-u_{n}\right)\right\}-m\left(u_{n}\right)\right] \\
-\lambda P_{C}\left[u_{n-1}-\rho E\left\{M_{n-1}+q+I\left(u_{n}-u_{n-1}\right)\right\}-m\left(u_{n-1}\right)\right]+(1-\lambda)\left(u_{n}-u_{n-1}\right),
\end{aligned}
$$

which can be written as, by using lemma 4.1 ,

$$
\begin{aligned}
& u_{n+1}-u_{n}-\lambda\left(m\left(u_{n}\right)-m\left(u_{n-1}\right)\right)-(1-\lambda)\left(u_{n}-u_{n-1}\right) \\
& <P_{C}\left[\{I-\rho E(M-1)\}\left(u_{n}-u_{n-1}\right)+\rho E L\left(u_{n+1}-u_{n}\right)-\left(m\left(u_{n}\right)-m\left(u_{n-1}\right)\right)\right]
\end{aligned}
$$

Again invoking lemma 4.1 and using the fact $\mathrm{P}_{\mathrm{C}}{ }^{2}=\mathrm{P}_{\mathrm{C}^{\prime}}$ we obtain

$$
\begin{gathered}
P_{C}\left[u_{n+1}-u_{n}-\lambda\left(m\left(u_{n}\right)-m\left(u_{n-1}\right)\right)-(1-\lambda)\left(u_{n}-u_{n-1}\right)\right]<\lambda P_{C}\left[\{I-\rho E(M-L)\}\left(u_{n}-u_{n-1}\right)\right. \\
\left.+\rho \operatorname{ELL}\left(u_{n+1}-u_{n}\right)-\left(m\left(u_{n}\right)-m\left(u_{n-1}\right)\right)\right]
\end{gathered}
$$

In a similar way, we have

$$
\begin{aligned}
& P_{C}\left[-\left\{u_{n+1}-u_{n}-\lambda\left(m\left(u_{n}\right)-m\left(u_{n-1}\right)\right)-(1-\lambda)\left(u_{n}-u_{n-1}\right)\right]\right. \\
& <\lambda P_{C}\left[-\{I-P E(M-L)\}\left(u_{n}-u_{n-1}\right)-P E L\left(u_{n+1}-u_{n}\right)+m\left(u_{n}\right)-m\left(u_{n-1}\right)\right] .
\end{aligned}
$$

Again using lemma 4.1 and adding (4.4) and (4.5), we obtain

$$
\begin{gathered}
\left|u_{n+1}-u_{n}-\lambda\left(m\left(u_{n}\right)-m\left(u_{n-1}\right)\right)-(1-\lambda)\left(u_{n}-u_{n-1}\right)\right|<\lambda\left|m\left(u_{n}\right)-m\left(u_{n-1}\right)\right| \\
+\lambda|I-\rho E(M-I)|\left|u_{n}-u_{n-1}\right|+\lambda \rho E|L|\left|u_{n+1}-u_{n}\right|,
\end{gathered}
$$




$$
\begin{aligned}
(I-\lambda \rho E|L|) \mid u_{n+1} & -u_{n}|<2 \lambda| m\left(u_{n}\right)-m\left(u_{n-1}\right)|+| I-\lambda \rho E(M-L)|| u_{n}-u_{n-1} \mid \\
& <\{2 \lambda N+|I-\lambda \rho E(M-L)|\}\left|u_{n}-u_{n-1}\right| \text {, by }(4.1) .
\end{aligned}
$$

Since $I$ is either a strictly upper or lower triangular matrix, the matrix $(I-\lambda \rho E|L|)$ is invertible for $0<\lambda<1$ and its inverse is non-negative, which implies that $(I-\lambda \rho E|L|)$ is an M-matrix. Hence

$$
\left|u_{n+1}-u_{n}\right|<(I-\lambda \rho E|L|)^{-1}[2 \lambda N+|I-\lambda \rho E(M-L)|]\left|u_{n}-u_{n-1}\right| \text {. }
$$

which is the required result (4.2). Ising similar arguments, we can obtained (4.3).

Fron theorem 4.1, we can establish a sufficient condition for the convergence of the sequence $\left\{u_{n+1}\right\}$ generated by algorithmm 3.3 to be bounded and hence have an accumulation point, which is the solution of the linear guasi complementarity problem $(2,9)$ and this is the main motivation of our next result.

THEOREM 4.2. Suppose that

$$
\sigma(G)<1 \text {, }
$$

where

$$
G=(I-\lambda \rho E|L|)^{-1}[2 \lambda N+|I-\lambda \rho E(M-L)|],
$$

with $\sigma$ denoting the spectral radius. Then for any initial vector $u$, the sequence $\left\{u_{n+1}\right\}$ generater by algorithm 3.3 converges to a solution of (2.9).

PROOF. The method proof is similar to that of Pang [14] and Noor and zarae [20]. We observe that the matrix $G$ defined by $(4.6)$ is non-negative. Hence from theorem 4.1 , we have

$$
\left|u_{n+1}-u_{n}\right|<G\left|u_{n}-u_{n-1}\right|
$$

Since $\sigma(G)<1$, it follows that

$$
\lim _{n \rightarrow \infty}\left|u_{n+1}-u_{n}\right|=0
$$

Next, by inductive arguments, we deduce that

$$
\begin{aligned}
\left|u_{n+1}-u_{n}\right| & <\sum_{i=0}^{i+1} G^{i}\left|u_{1}-u_{0}\right| \\
& <(1-G)^{-1}\left|u_{1}-u_{0}\right|
\end{aligned}
$$

where the last inequality follows from the fact that the matrix $G$ is non-negative and $\left.\sigma^{\star} G\right)<1$, see $[40]$. Hence we conclude that the sequence $\left\{u_{n+1}\right\}$ is bounded and has an accumulation point, say $u^{\star}$. Let $\left\{u_{n_{i}+1}\right\}$ be subsequence convering to $u^{\star}$. Then from (4.7) we see that $\left\{u_{n_{i}+1}\right\}$ converges to $u^{*}$ as well. Since the mappings are continuous, so by passing to the limit $n_{i} \rightarrow \infty$, we obtain

$$
u^{\star}=(1-\lambda) u^{\star}+\lambda m\left(u^{\star}\right)+\lambda P_{C}\left[u^{*}-p E\left(M u^{*}+q\right)-m\left(u^{*}\right)\right]
$$


which is eruivalent to quasi conplenentarity problek (2.9) by lenma 3.1 and lenma 3.2 , that is $11^{*} i$; the solution of quasi complementarity problem (2.9). We finally show that the sequence $\left\{u_{n+1}\right\}$ zonverges to $u^{*}$. From (4.3), we obtain

$$
\left|u_{n+1}-u^{*}\right|<g\left|u_{n}-u^{*}\right|
$$

where $G$ is as defined by $(4.6)$. Since $\sigma(G)<1$, it follows that the entire sequence $\left\{u_{n+1}\right\}$ converyes $t_{1} u^{*}$, and this completes the proof of the theorem 4.?.

We note that for $\lambda=1$, these results reduce to the earlier results obtained in [20]. Also it is clear from theorem 4.2, that the condition $\sigma(G)<1$ provides existence an. 1 uniqueness result for the linear quasi complementarity problem (2.9). Note also that the matrix $M$ is not assumed to be symmetric.

If the mapping $\mathrm{m}$ is zero, then the non-negative matrix $\mathrm{N}$ becomes the zero matrix. Consequently our results reduce to the results of Ahn [22]. Thus we conclude that the algorithms of the Mangasarian types can be extended to study the linear quasi complementarity problem. The results proved here are an improvement as well as extension of the ones obtained by Ahn $[21,22]$, Mangasarian [19], Pany [14,15] and Noor and Zarae [20] for certain special cases.

In order to discuss the usefulness of theorem 4.2 , we need the following concept.

Definition 4.1. If $B$ is an invertible matrix, $B^{-1}>0$ and $C>0$, then $A=B-C$ is known as regular splitting of $A$.

Now let

and

$$
B=I-\lambda \rho E|L|
$$

$$
C=2 \lambda N+|I-\lambda \rho E(M-L)|
$$

It is clear that the matrix $B$ is invertible and $B^{-1}>0$. Furthermore, $C>0$. Hence from (4.5) and a result of ortega and Rheinboldt [40], we obtain

$$
\sigma(G)=\sigma\left(B^{-1} C\right)<1
$$

if and only if $A^{-1}$ exists and non-negative.

Note that

$$
A=B-C=1-\lambda \rho E|L|-2 \lambda N-|I-\lambda \rho E(M-L)|
$$

is a z-matrix. If the matrix $A$ is a P-matrix, then it follows that $A$ has nonnegative inverse.

Frorn the above discussion, we obtain the following results, which appears to be new ones.

Corollary 4.1. If $A=I-|I-\lambda \rho E D|-\lambda \rho E(|L|+\mid K+U-L) \mid)-2 \lambda N$, where $M=K+D+U$, is a P-matrix, then the sequence $\left\{u_{n+1}\right\}$ generated by algorithm 3.2
with $L=K, U$, or 0 and 


$$
\left.0<\lambda \rho<\frac{1+2 \lambda N}{\max \left(M_{j j}\right.} E_{j j}\right)
$$

converges to a solution of the linear quasi complementarity problem (2.9).

For $\lambda=1, C=R_{+}^{n}$, and the point-to-point mapping $m$ equal to zero, all our results reduce ts the results of Ahn [21].

Froin now onwarl, we shall assume that

$\mathrm{Y}=\mathrm{K}+\mathrm{D}+\mathrm{U}$,

where $D$ is diagonal (not necessarily positive). $K$ is strictly lower triangular and $U$ is strictly upper triangular and the point-to-point mapping $m$ is zero. We now give some specific cases of the fundamental and general algorithm 3.2 .

By taking $\mathrm{L}=0$, in algorithm 3.2, we obtain the following algorithms, which are agains new ones.

Algorithm 4.1. (Projected Jacobi over relaxation).

Iset $u_{0} \varepsilon C$, then

$$
u_{n+1}=(1-\lambda) u_{n}+\lambda P_{C}\left[u_{n}-\rho E\left(M_{n}+q\right)\right], \quad n=0,1,2, \ldots
$$

where $0<\lambda<1, \rho>0$ and $E$ is a positive diagonal matrix such that $2(\lambda \rho E)^{-1}-M$ is positive definite.

Note further that, if $D$ is a positive diagonal matrix, $E$ may be taken equal to $D^{-1}$, as in the case of Jacobi over relaxation method. If in addition, $\lambda=\rho=1$, we obtain the projected ordinary Jacobi iteration.

By taking $\mathrm{I}=\mathrm{K}$ or $\mathrm{U}$, in algorithm 3.2, where $\mathrm{E}$ is a positive diagonal matrix, we obtain the following algorithm.

Algorithm 4.2. (projected SOR)

$$
\begin{aligned}
& \text { Let } u_{0} \varepsilon C \text {, compute } \\
& u_{n+1}=(1-\lambda) u_{n}+\lambda P_{C}\left[u_{n}-\rho E\left(M_{n}+q+L\left(u_{n+1}-u_{n}\right)\right)\right], \quad n=0,1,2, \ldots
\end{aligned}
$$

where

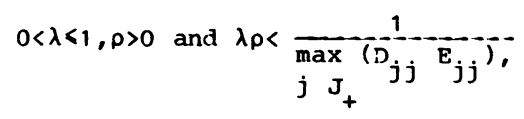

with $J_{+}=\left\{j: D_{j j}>0, \quad j=1,2, \ldots\right\}$

Remark 4.2. For $\lambda=1, E=D, L=K, C=R$, and the mapping $m$ equal to zero, algorithm 3.2 becomes the projected SOR method studied by cryer, Eckhardt and many others. If in adition $\rho=1$, algorithm 3.2 becomes the projected Gauss-seidel method.

Remark 4.3. It is evident that the convergence analysis of the algorithm $3.3 \mathrm{holds}$ only for $c=[0, b]$. The question arises can this restriction be relaxed. The answer 
to this is partly true. Indeed, this is true for the nonlinear quasi conplementarity problem as shown below. For the linear complementarity problem, however, the question still reinains open.

It has been shown by pang [14] that the condition 4.1 for the point-to-point mapping $m$ is equivalent to the fact the mapping $m$ is Lipschitz continuous, that is there exists a $\gamma>0$ such that

$$
\| m(u)-m(v)||<y|| u-v||, \quad \text { for all } u, v \text {. }
$$

We also need the following concepts:

Definitions 4.1. A mapping $T ; R^{n} \longrightarrow R^{n}$ is said to be

i). Stronyly Monotone, if there exists a constant $\alpha>0$ such that

$$
(T u-T v, u-v)>\alpha|| u-v \mid \|^{2}, \quad \text { for all } u, v \in R^{n}
$$

ii). Lipschitz continuous, if there exists a constant $B>0$ such that

$$
\|\mathrm{Tu}-\mathrm{Tv}\|<\beta|| \mathrm{u}-\mathrm{v} \|, \quad \text { for all } \mathrm{u}, \mathrm{v} \in \mathbf{R}^{\mathbf{n}} \text {. }
$$

In particular, it follows that $\alpha<\beta$.

In the next theorem, we study the conditions under which the approximate solution obtained froin (3.3) converges to the exact solution $u$ of nonlinear quasi complementarity problem $(2.8)$. This result shows that the convergence of the approximate solution to the exact solution also depends on the relaxation parameter $\lambda$ used after projection like the linear quasi complementarity problem. Furthermore, we prove that the restriction $C$ is a closed rectangle in $R^{n}$ can be relaxed for nonlinear quasi complementarity problem and the convergence analysis hold for any general closed convex set $C$ in $R^{n}$. The next result is an improvement of a result of Noor [16].

THEOREM 4.3. Let the continuous mapping $T$ from $R^{n}$ into itself be strongly monotone and Lipschitz continuous. If the mapping $m$ is also Lipschitz continuous with Lipschitz consttant $\gamma$ and $u_{n+1}$ and $u$ are solutions satisfying $(3.3)$ and $(2.8)$, then

$$
u_{n} \longrightarrow u \text { in } R^{n} \text {, }
$$

for $\quad\left|\rho-\frac{\alpha}{\beta^{2}}\right|<\frac{\sqrt{\alpha^{2}-4 \beta^{2}\left(\gamma-\gamma^{2}\right)}}{\beta^{2}}, \quad \alpha>2 \beta \sqrt{\gamma-\gamma^{2}}, \quad \gamma<\frac{1}{2} \quad$ and $\quad \lambda<1 /\left[1-2 \gamma-\sqrt{\left.1-2 \rho \alpha+\rho^{2} \beta^{2}\right]}\right.$.

PROOF. From lemmas 3.1 and 3.2 , we conclude that the solution $u$ of $(2.8)$ can be characterized by the solution (3.3). Hence from (3.2) and (3.3), we have

$$
\begin{aligned}
& || u_{n+1}-u||<|| \lambda m\left(u_{n}\right)+\lambda P_{C}\left[u_{n}-\rho T\left(u_{n}\right)-m\left(u_{n}\right)\right]+(1-\lambda) u_{n} \\
& -\lambda m(u)-\lambda P_{C}[u-\rho T(u)-m(u)]+(1-\lambda) u|| \\
& <\lambda|| m\left(u_{n}\right)-m(u)||+\lambda|| u_{n}-u-p\left(T\left(u_{n}\right)-T(u)\right)-m\left(u_{n}\right)+m(u)|| \\
& +(1-\lambda)\left\|u_{n}-u\right\|, \quad \text { since } P_{C} \text { is a non-expansive [5]. } \\
& <2 \lambda|| m\left(u_{n}\right)-m(u)||+|| u_{n}-u-p\left(T\left(u_{n}\right)-T(u)\right)||+(1-\lambda)|| u_{n}-u|| \text {. }
\end{aligned}
$$


Now by the strongly monotonicity and Lipschitz continuity of $T$, we have

$$
|| u_{n}-u-\rho\left(T\left(u_{n}\right)-T(u)\right)||^{2}<\left(1-2 \alpha \rho+\beta^{2} \rho^{2}\right)|| u_{n}-u \|^{2} \text {. }
$$

Thus using the above inequality and Lipschitz continuity of in, we obtain

$$
\begin{aligned}
& \begin{aligned}
\| u_{n+1}-u|| & <\left(2 \lambda \gamma+(1-\lambda)+\lambda \sqrt{1-2 \alpha \rho+\rho^{2} \beta^{2}}\right) \| u_{n}-u||, \\
& =\theta|| u_{n}-u||,
\end{aligned} \\
& \text { where } \theta=2 \lambda \gamma+(1-\lambda)+\lambda \sqrt{1-2 \alpha \rho+\beta^{2} \rho^{2}}=2 \lambda \gamma+(1-\lambda)+\lambda t(\rho), \text { with } t(\rho)=\sqrt{1-2 \alpha \rho+\beta^{2} \rho^{2}}
\end{aligned}
$$

Now $t(\rho)$ assumes its minimum value for $\bar{\rho}=\frac{\alpha}{\beta^{2}}$ with $t(\bar{\rho})=\sqrt{1-\frac{\alpha^{2}}{\beta^{2}}}$. We have to show that $\theta<1$, if $\rho$ satisfies $2 \lambda \gamma+(1-\lambda)+\lambda t(\rho)<1$, which reduces to $2 \gamma+t(\rho)<1$. For $\rho=\bar{\rho}, \quad 2 \gamma+t(\rho)<1$ implies that $\gamma<\frac{1}{2}$ and $\alpha>2 \beta \sqrt{\gamma-\gamma^{2}}$. Thus it follows that $\theta=2 \lambda \gamma+(1-\lambda)+\lambda t(\rho)<1$ for all $\rho$ with

$$
\left|\rho-\frac{\alpha}{\beta^{2}}\right|<\frac{\sqrt{\alpha^{2}-4 \beta^{2}}\left(\gamma-\gamma^{2}\right)}{\beta^{2}}, \alpha>2 \beta \sqrt{\gamma-\gamma^{2}} \text { and } \gamma<\frac{1}{2} \text {. }
$$

Also $\theta>0$ leads to the requirement that $\lambda<1 /\left[1-2 \gamma-\sqrt{\left.1-2 \alpha \rho+\beta^{2} \rho^{2}\right]}\right.$.

Since $\theta<1$, so the fixed problem (3.2) has a unique solution $u$ and consequently, the iterative solution $u_{n+1}$ obtained from (3.3) converges strongly to $u_{\text {, the exact }}$ solution of nonlinear quasi complementarity problem (2.8), which is the required result.

\section{Special Cases 4.1 .}

i) It is also evident from the proof of theorem 4.3 that the convergence criteria depends on the relaxation factor $\lambda$ used after the projection.

ii) If the point-to-point mapping $m$ is zero, then theorem 4.3 is exactly the same as proved in Noor $[41]$.

iii) The case $\lambda=1$ is considered by Noor [32].

iv) If the point-to-point mapping $m$ is zero, that is the Lipschitz constant $Y$ is zero, then for $\lambda=1$, theorem 5.5 is exactly the same as proved by Fang [35] and Aganaqic [33]. For related work, see Pang and Chan [42].

CONCLUION. The algorithms suggested in this paper are the most general and unifying ones. The results proved in this paper are an improvement as well as extension of the previous ones for the complementarity problems. We have shown only the possibility that the iterative algorithms such as Algorithms 3.1 and 3.2 can solve the nonlinear and linear quasi complementarity problems. Development and improvement of implementable algorithms for this class of problems deserves further research efforts. 
ACKNOWLEDGEMENTS. The author is grateful to the referee for his helpful suggestions and cominents. This researsh is supported by the Research centre of college of Scipnce, king saud University under grant No. Math/14J5/15.

\section{REFERENCES}

1. COTTLE, R.W. and DANTZIG, G.B. Complementarity pivot theory of mathematical proqranming, Linear Alg. Appl. 1(1968), 163-185.

2. LEMKE, G.E. Recent results on complementarity problems in Nonlinear proyranming, (ed.) by H.R. Rosen, O.L. Mangasarian and K. Ritter, Academic Press, 1970 .

3. BENSOUSSAN, A. and LIONS, J. Applications des Inequations variationelles en control and stoschastiques, Dunod, paris, 1978 .

4. CRANK, J. Free and moving boundary problems, Oxford University Press, U.K. 1984.

5. BAIOCCHI, C. and CAPELO, A. Variational and quasi variational inequalities, J. Wiley and Sons, New York, Jondon, 1984 .

5. ODEN, J.T. and KIKUCHI, N. Theory of variational inequalities with applications to problems of flow through porous media, Int. J. Engng. Sci. 18(1980), 1173-1284.

7. HABETLER, G.H. and PRICE, A.L. Existence theory for generalized nonlinear complementarity problems, J. Opt. Th. Appl. I(1971), 223-239.

8. HABETLER, G.H. and PRICE, A.L. An iterative method for generalized nonlinear complementarity problems, J. Opt. Th. Appl. 11(1973), 36-48.

9. KARAMARDIAN, S. Generalized complementarity problems, J. Opt. Th. Appl.: $8(1971)$, 223-239.

10. DOLCETTA, I. Sistemi di complementarita a diseguaglianze variationali, Ph.D. Thesis, Jniversity of Rome, Italy, 1972 .

11. LIONS, J. optimal control of systems governed by partial differential equations, Springer-Verlag, New York, Berlin, 1971.

12. MANCINO, O. and STAMPACCHIA, G. Convex programming and variational inequalities, J. Opt. Th. Appl. $9(1972), 3-23$.

13. KARAMardia, S. The complementarity problem, Math._Prog. 2(1972), 107-129.

14. PANG, J.S. On the convergence of a basic iterative method for the implicit complementarity problem, J. Opt. Th. Appl. 37(1982), 149-162 .

15. PANG, J.S. The implicit complementarity problem in Non-linear programming (ed.) by O.L. Mangasarian, R. Meyer and S.M. Robinson, Academic Press, New York, Jondon, 4(1981), 487-518.

15. NOOR, M.A. Generalized quasi complementarity problems, J. Math. Anal. Appl. $120(1986), 321-327$.

17. NOOR, M.A. An iterative scheme for a class of quasi variational inequalities, J. Math. Anal.Appl. 110(1985), 463-468.

18. CHAN, D. and PANG, J.S. The generalized quasi variational ineguality problem, Math. Oper. Res. 그. 1982), 211-222.

19. Mangasarian, O.L. Solution of symmetric linear complementarity problems by iterative methods, J. Opt. Th. Appl. 22(1977), 465-485.

2n. NOOR, M.A. and ZARAE, S. Linear quasi complementarity problems, Jutilitas Math. 27(1985), 249-260.

21. AHN, H.B. Solutions of nonsymmetric linear complementarity problems by iterative methods, J. Opt. Th. Appl. 33(1981), 175-185. 
22. AHN, H.B. Iterative mothods fir linear conplementarity problems with upper bounds on primary variables, Math. prog. 26(1983), 295-315.

23. STAMPACCHIA, G. Formes bilineaires coercives sur less ensembles convexes, C.R. Acad. Sci. Paris, 285(1964), 4413-4416.

24. FICHERA, G. Problemi elastostaici con vincoli unilaterali; et problema di Signorini con ambiqne conditizioni al contorno, Atti. Acad. Naz. Lincei Mem. Cl. Sci. Fis. Mat. Nat. Sez. Ia(8), VII, (1964), (1966), 71-140.

25. MOSCO, U. and STRANG, G. One sided approximation and variational inequalities, Bull. Amer. Math. Soc. 80(1974), 308-312.

25. NOOR, M.A. On variational inequalities, Ph.D. Thesis, Brunel University, J.K. 1975 .

27. LIONS, J. and STAMPACChIA, G. Variational inequalities, Comm. Pure Appl. Math. 20(1967), 493-519.

28. GLOWINSKI, R., LIONS, J. and TREMOLIERES, R. Numerical analysis of variational inequalities, Nor th-Holland, 1982 .

29. NOOR, K.I. and NOOR, M.A. Iterative methods for a class of variational inequalities in Numerical Analysis of Singular Perturbation problems (ed.) by J. Miller and $\vec{w}$. Hemker, Academic Press, London, New York, (1979), 441-449.

30. NOOR, M.A. and NOOR, K.I. Iterative methods for variational inequalities and nonlinear programming, Op. Res. Verf. 31(1979), 455-463.

31. Mosco, U. Implicit variational problems and quasi variational inequalities, Lect. Notes Math. 543, Springer-Verlag, 1976.

32. NOOR, M.A. Iterative methods for quasi complementarity problems, Methods Op. Res. 56 1986$), 75-83$.

33. AGANAGIC, M. Variational inequalities and generalized complementarity problems, Technical Rep. SOL. 78-11, Department of operations Research, stanford University, J.S.A., 1978 .

34. COTTLE, R.W. Complementacity and variational problems, symp. Math. 19(1976), 177-208.

35. FANG, S. An iterative method for generalized complementarity problems, IEEE Trans. Aut. Control. 25(1,980), 1225-1227.

36. NOOR, M.A. Strongly nonlinear variational inequalities, C.R. Math. Rep. Acad. Sci. Canada, $\underline{4}(1982), 213-218$.

37. CRYER, C.W. The solution of a quadratic programming problem using systematic over relaxation, SIAM_J.-Control. 9(1971), 385-392.

38. COTTLE, R.W. and GOHEEN, M. A special class of large quadratic programs in Nonlinear programming, (ed.) by O.L. Mangasarian, R. Meyer and S.M. Robinson, Academic Press, 3(1978), 361-390.

39. VARGA, R.S. Matrix iterative analysis, prentice-Hall, New Jersey, U.S.A., 1961.

40. ORTEGA, J.M. and RHEINBOLDT, W.C. Iterative solutions of nonlinear equations in several variables, Academic Press, New York, London, 1970 .

41. NOOR, M.A. Generalized nonlinear complementarity problems, J. Nat. Sci.\& Math. 26 $(1986), 9-20$.

42. PANG, J.S. and CHAN, D. Iterative methods for variational and complementarity problems, Math.Prog., 24 (1982), 284-313. 


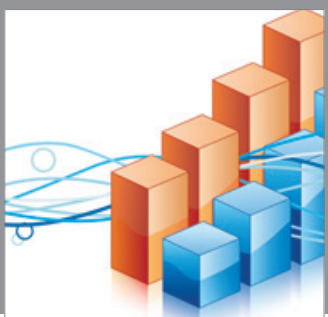

Advances in

Operations Research

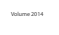

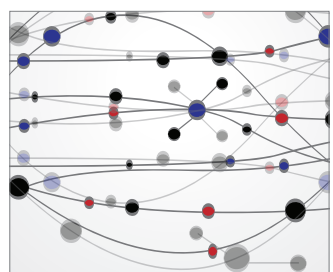

\section{The Scientific} World Journal
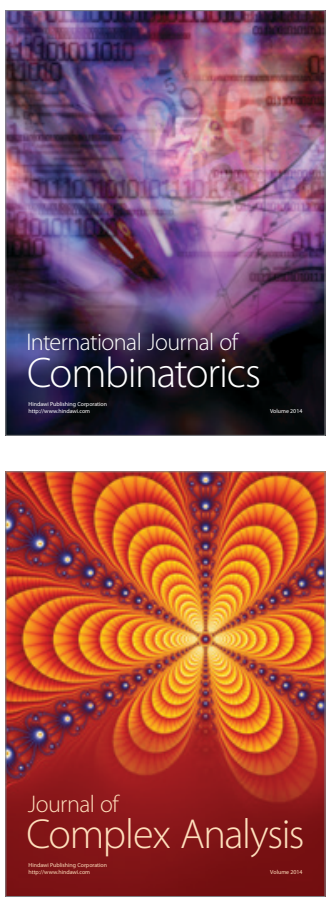

International Journal of

Mathematics and

Mathematical

Sciences
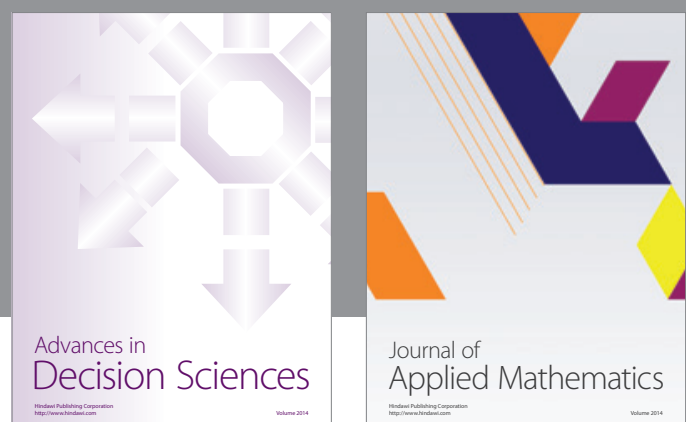

Journal of

Applied Mathematics
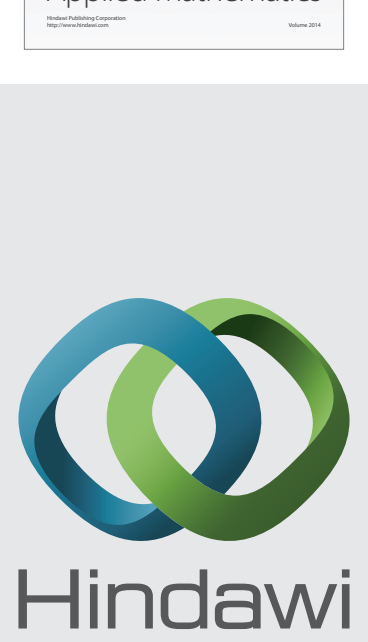

Submit your manuscripts at http://www.hindawi.com
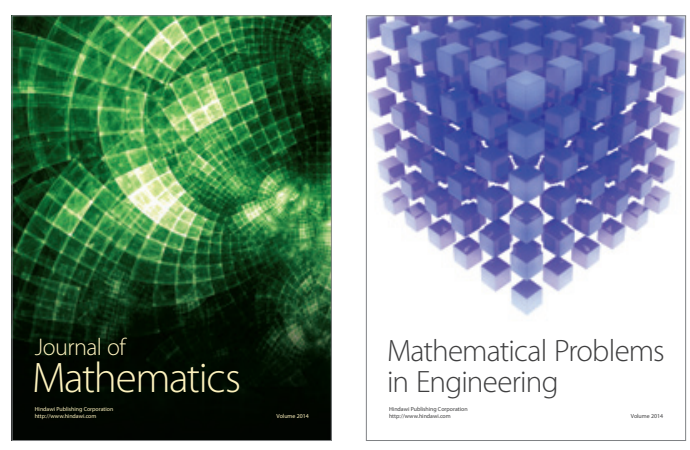

Mathematical Problems in Engineering
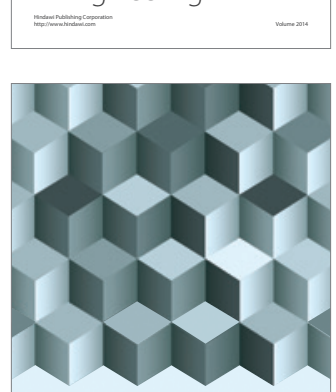

Journal of

Function Spaces
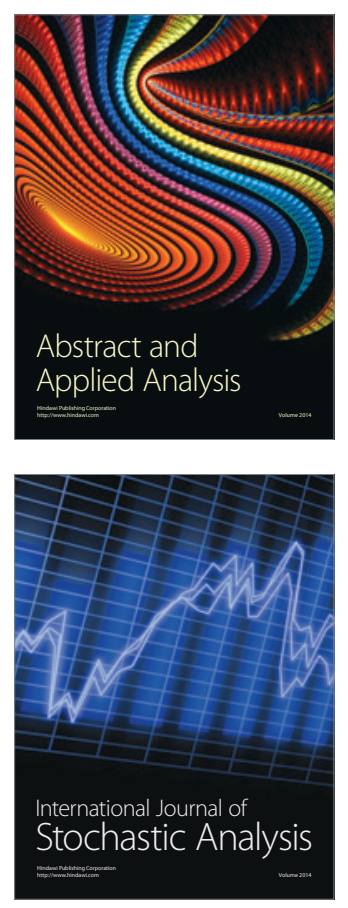

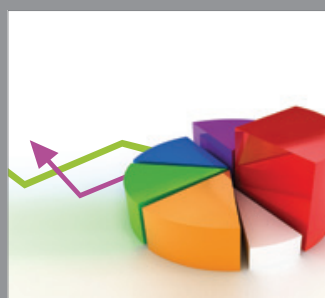

ournal of

Probability and Statistics

Promensencen
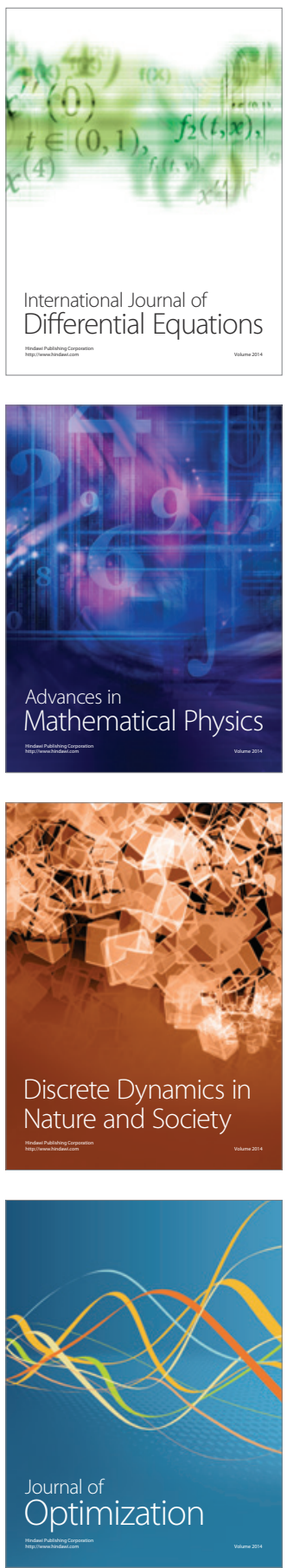